\title{
Algoritmo de Alocação de Blocos de Recurso em Redes CP-OFDM com Características 5G Baseado em Estimação de Retardo
}

\author{
Marcus V. G. Ferreira ${ }^{1}$, Flávio H. T. Vieira ${ }^{1}$ \\ ${ }^{1}$ Instituto de Informática - Universidade Federal de Goiás (UFG) \\ Alameda Palmeiras, Quadra D, Câmpus Samambaia - 74690-900 - \\ Goiânia - GO - Brazil \\ marcusferreira@inf.ufg.br, flavio_vieira@ufg.br
}

\begin{abstract}
In this paper, a resource allocation algorithm for wireless communication systems is proposed, which considers the channel transmission quality and data delay. In the proposed approach, the delay value is estimated to decide on the allocation of available radio resources, aiming to reduce the system's average delay. For the delay estimation, an approach involving concepts of Network Calculus such as service curve and envelope process MFBAP (Multifractal Bounded Arrival Process) is proposed. In view of the recent techniques for $5 G$ communication systems, $\mathrm{mmWa-}$ ves propagation, 256-QAM (Quadrature Amplitude Modulation) and carrier aggregation technologies are considered. Simulations are carried out to compare the performance of the proposed resources allocation algorithm with other algorithms in the literature in terms of QoS (Quality of Service) parameters such as delay, throughput, processing time, loss rate and fairness index, verifying the efficiency of the proposed algorithm.
\end{abstract}

Resumo. Neste artigo, é proposto um algoritmo de alocação de recursos para sistemas de comunicação sem fio que considera a qualidade de transmissão do canal e o retardo dos dados. Nesta proposta, o valor do retardo é estimado para decidir sobre a alocação dos recursos de rádio disponíveis, objetivando reduzir o retardo médio do sistema. Para a estimativa do retardo, é proposta uma abordagem envolvendo conceitos de Cálculo de Rede como curva de serviço e processo envelope MFBAP (Multifractal Bounded Arrival Process, Processo de Chegada Limitado Multifractal). Tendo em vista as recentes técnicas para sistemas de comunicação 5G, são consideradas as tecnologias de propagação por ondas milimétricas (mmWaves), 256-QAM (Quadrature Amplitude Modulation, Modulação de Amplitude em Quadratura) e agregação de portadora. As simulações são desenvolvidas para comparar o desempenho do algoritmo de alocação de recursos proposto com demais algoritmos da literatura em termos de parâmetros de QoS (Quality of Service, Qualidade de Serviço) como retardo, vazão, tempo de processamento, taxa de perda e indice de justiça, verificando a eficiência do algoritmo proposto. 


\section{Introdução}

Na literatura da área de comunicações móveis há várias propostas de esquemas de alocação de recursos em redes sem fio [Su et al. 2012] [Guan et al. 2011] [Ferreira et al. 2015]. Em [Su et al. 2012], é proposto um algoritmo para alocação de blocos de recursos em redes sem fio baseado na heurística PSO (Particle Swarm Optimization, Otimização por Enxame de Partículas), com o objetivo de maximizar a vazão total do sistema. Em [Guan et al. 2011], é proposto um algoritmo com o objetivo de garantir o critério de taxa mínima de transmissão requerida pelo usuário. Em [Ferreira et al. 2015], é proposto um algoritmo com o objetivo de minimizar o parâmetro de retardo e atender critérios de QoS (Quality of Service, Qualidade de Serviço). Estes esquemas de alocação, em adição a outros não mencionados, tem o mesmo propósito de maximizar a vazão total através de heurísticas que focam em diferentes parâmetros de QoS como retardo, taxa mínima, dentre outros.

Neste artigo, é proposto um algoritmo que tem como objetivo reduzir o retardo do sistema, parâmetro de QoS essencial para aplicações em tempo real com taxa de transmissão variável e requisitos específicos de banda, como serviços de VoIP (Voice over Internet Protocol, Voz sobre Protocolo Internet) e de videoconferência. O limitante de retardo é estimado através do Cálculo de Rede Determinístico a fim de prover QoS em redes de comunicação, diferentemente de outros trabalhos da literatura que utilizam valores reais passados [Ferreira et al. 2015] [Delgado and Jaumard 2010] de retardo da rede. Assim, na proposta apresentada, pode-se atualizar a estimativa de retardo a medida que as características dos dados de tráfego no sistema variam. Na abordagem proposta de estimação de retardo são utilizados conceitos de curva de serviço e processo envelope MFBAP (Multifractal Bounded Arrival Process, Processo de Chegada Limitado Multifractal) [Santos Jr and Vieira 2015].

Tendo em vista os requisitos para suportar a geração de redes sem fio $5 \mathrm{G}$, são cobertas várias características destas neste artigo, com conceitos como agregação de portadora, 256-QAM (Quadrature Amplitude Modulation, Modulação de Amplitude em Quadratura), estrutura de radio com espaçamento entre subportadoras de 120 $\mathrm{KHz}$ e, por fim, cenário com ondas milimétricas acima de $6 \mathrm{GHz}$ [3GPP 2017] [3GPP 2018b] [3GPP 2018a] [3GPP 2019]. Todos estes recursos possibilitam prover ao usuário final maiores taxas de transmissão de dados assim como menores valores de retardo na rede.

O artigo está organizado da seguinte forma: na Seção 2 são apresentados os conceitos de Cálculo de Rede, processo envelope MFBAP, curva de serviço e limitante de retardo; na Seção 3 é apresentado o modelo de transmissão downlink; na Seção 4 é proposto o algoritmo de alocação de blocos de recurso em redes sem fio; na Seção 5 são apresentados os parâmetros de simulação e os resultados; na Seção 6 é concluído o artigo com as considerações finais.

\section{Cálculo de Rede Determinístico}

O Cálculo de Rede Determinístico pode ser utilizado para estimar recursos a fim de prover QoS em redes e tem fornecido ferramentas poderosas para estimação do backlog e retardo em uma rede com garantia de serviço para fluxos de tráfego indivi- 
duais. Usando a noção de processo envelope, curvas de chegada e curvas de serviço, vários trabalhos tem demonstrado que os limitantes de backlog e retardo podem ser concisamente expressos pela álgebra Min-Plus [Le Boudec and Thiran 2004].

O Cálculo de Rede também pode ser visto como a teoria de sistemas que se aplica às redes de computadores, mas a principal diferença é considerar-se outra álgebra, onde as operações são alteradas da seguinte forma: adição torna-se o cálculo do mínimo, e a multiplicação torna-se adição.

\subsection{Processo envelope MFBAP}

O processo envelope para o tráfego de chegada de pacotes é um limitante superior para o processo real de tráfego de pacotes acumulados. Para um processo envelope determinístico, a função limitante $\hat{A}(t)$ corresponde ao valor máximo de um fluxo $A(t)$ no intervalo de tempo $[s, s+t$ ], e é definida pela equação [Le Boudec and Thiran 2004]:

$$
\hat{A}(t)=\sup _{s \geq 0} A[s, s+t],
$$

onde sup é um operador que retorna o valor máximo de $A[s, s+t]$ sem estabelecer valor limite superior, sendo $s \geq 0$ neste caso.

O MFBAP é uma alternativa determinística de se obter o processo envelope que limita o volume do tráfego em um dado intervalo de tempo, calculado da seguinte forma [Santos Jr and Vieira 2015]:

$$
\hat{A}_{M F B A P}(t)=\bar{a} t+k \sigma t^{H(t)}+B,
$$

onde $H(t)$ é o expoente de Hölder [P et al. 2003], que representa o grau da singularidade da função, $t$ é o instante de tempo, $\bar{a}$ e $\sigma$ são respectivamente, a média e o desvio padrão do tráfego de entrada, $k$ é a constante relacionada à probabilidade de violação (para $\epsilon=10^{-6}$ ) do processo envelope e $B$ é o tamanho do buffer.

\subsection{Estimativa de limitante de retardo}

O limitante superior de retardo estimado, denotado por $\widehat{d}$, é dado por [Santos Jr and Vieira 2015]:

$$
\widehat{d}=\inf \left\{d \geq 0 \mid \forall t \geq 0: A^{*}(t-d) \leq S(t)\right\},
$$

onde inf é um operador que retorna, neste caso, o menor valor de $d \geq 0$ que atende $A^{*}(t-d) \leq S(t)$.

Assim, propõe-se neste artigo utilizar essa equação para estimar o retardo, onde $A^{*}$ é o processo envelope MFBAP, calculado conforme Equação (2), e $S$ é a curva de serviço do sistema. Considerando um sistema CP-OFDM (Cyclic Prefix - Orthogonal Frequency Division Multiplexing, Prefixo Cíclico - Multiplexação por Divisão de Frequência Ortogonal) generalizado para qualquer usuário servido pelo mesmo intervalo de tempo $T$, sua curva de serviço pode ser escrita como [Vieira et al. 2016]:

$$
S_{n}(t)=c T P+c T \min \left\{\frac{\max [t-P N T-(n-1) T ; 0]}{T} ; 1\right\},
$$


onde $c$ é a média da taxa de atendimento no servidor do sistema e $N$ é o número de intervalos de tempo $T$ por ciclo completo $P$ dado por $P=\left\lfloor\frac{t}{N T}\right\rfloor$, onde o operador representa o menor número inteiro mais próximo de $\frac{t}{N T}$.

A Figura 1 mostra os valores médios de retardo estimados dos dados dos usuários conforme Equação (3) e os valores de retardo real observados nas simulações para diferentes distâncias considerando o escalonamento Round-Robin e o escalonamento proposto neste artigo para cenários distintos de uma rede sem fio CP-OFDM com largura de banda de $10 \mathrm{MHz}$. Os demais parâmetros de simulação do sistema de transmissão sem fio e da modelagem do canal são descritos na Tabela 1 .

Observa-se que os valores estimados são em geral similares aos valores de retardo do algoritmo proposto neste artigo, um pouco superiores se considerado até 5 usuários em simulação, enquanto os valores de retardo com escalonamento RoundRobin são em geral superiores aos demais. O uso desta técnica de estimativa de limitante de retardo foi exaustivamente testado em simulações com diversos cenários e se provou uma estratégia acertada tendo em vista a eficiência em representar o retardo da rede e os resultados obtidos.

\section{Modelo de transmissão downlink}

\subsection{Estrutura do frame de rádio}

A estrutura de um frame de transmissão downlink é mostrado na Figura 2. Cada frame de rádio ocupa $10 \mathrm{~ms}$, que são divididos em dez subframes de $1 \mathrm{~ms}$ [Guan et al. 2011]. Cada subframe por sua vez é dividido em dois slots de tempo de 0.5 ms. Há sete ou seis símbolos para cada slot de tempo, dependendo da utilização de, respectivamente, prefixo ciclo (CP, Cyclic Prefix) normal ou estendido [Guan et al. 2011].

No domínio da frequência os recursos são agrupados em 12 subportadoras com largura de banda entre 15 e $240 \mathrm{KHz}$ cada, conforme configuração da rede [3GPP 2018b]. Um RB (Resource Block, Bloco de Recurso) é definido como uma unidade de 12 subportadoras durante um slot de tempo [3GPP 2008], e estes são escalonados sempre em pares de RBs, chamados assim de blocos de escalonamento (SB, Scheduling Block), com duração de $1 \mathrm{~ms}$.

\subsection{Modelo do sistema}

Considerando um cenário de transmissão downlink de um sistema com uma antena, $N$ blocos de recursos disponíveis por TTI (Transmission Time Interval, Intervalo de Tempo de Transmissão), quantidade de potência distribuída igualmente entre todas as subportadoras e $K$ usuários servidos a taxas mínimas $R_{k}$. Um bloco de recurso é definido como $N_{s}$ símbolos consecutivos no domínio do tempo e $N_{s c}$ subportadoras no domínio do tempo. Considerando que existem sinais pilotos e de controle nos blocos de recursos, apenas $N_{s c}^{d}(s)$ subportadoras podem ser utilizadas para transferência de dados no $s$-ésimo símbolo, onde $s \in\left\{1,2, \ldots, N_{s}\right\}$ e $N_{s c}^{d}(s) \leq N_{s c}$. Seja $R_{j}^{(c)}$ a taxa de código associada ao MCS (Modulation and Coding Scheme, Esquema de Modulação e Codificação) $j \in\{1,2, \ldots, J\}$, onde $J$ é o número total de MCS suportados, $M_{j}$ o 


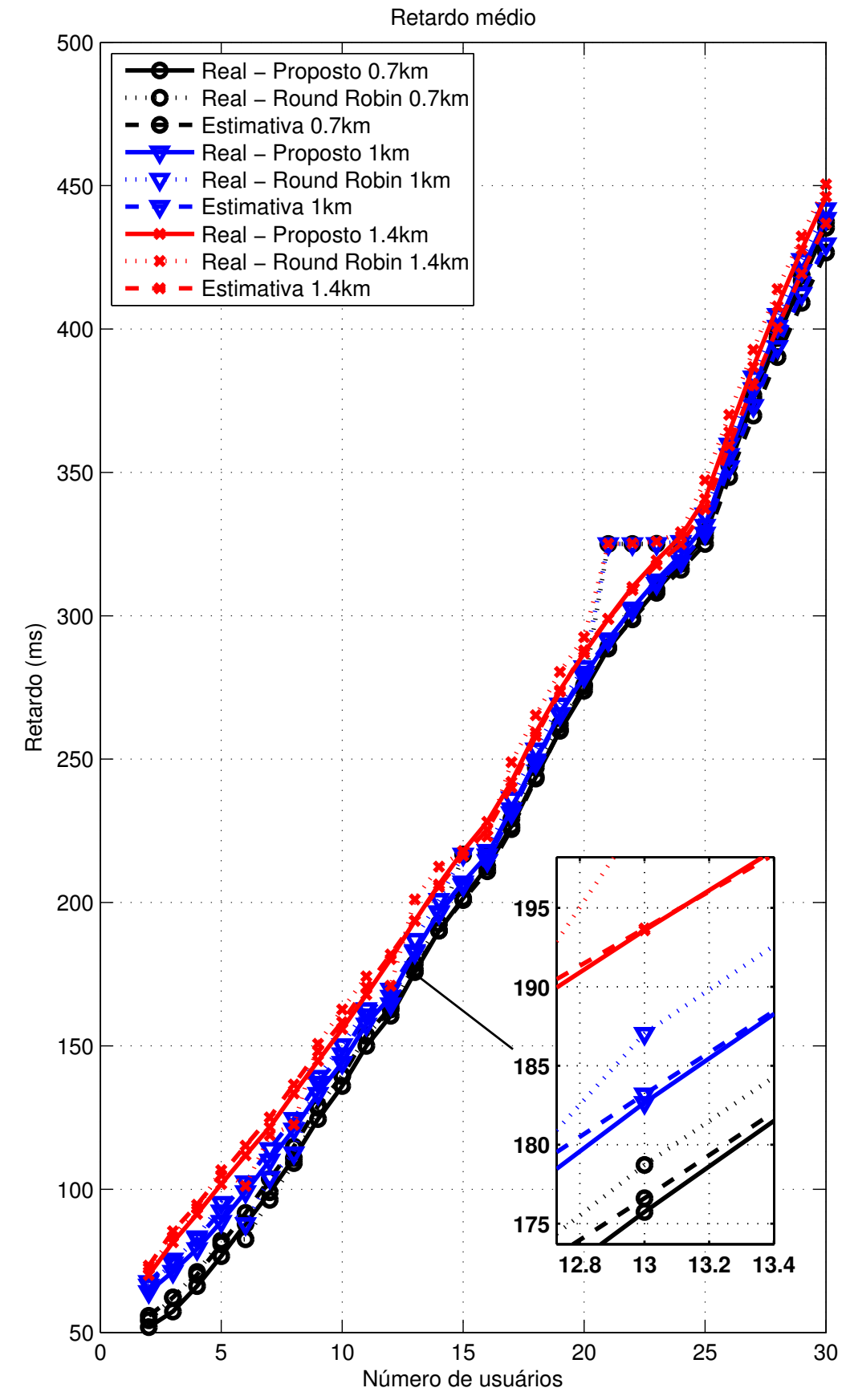

Figura 1. Valores médios de retardo estimado e real para o escalonamento RoundRobin e escalonamento proposto, considerando cenários com distância entre UE (User Equipment) e BS (Base Station) de 0.7, 1 e 1.4 km 


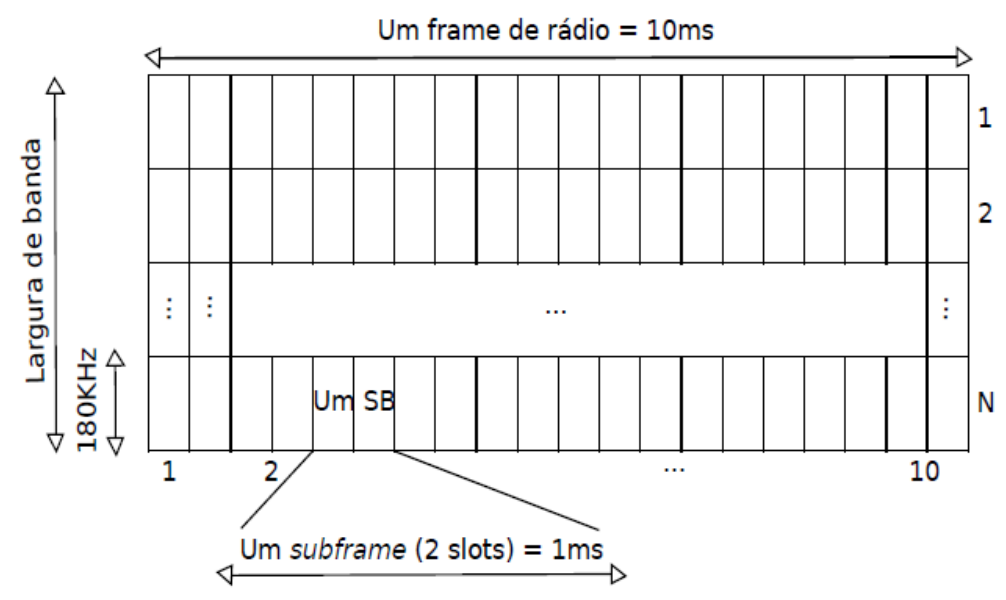

Figura 2. Estrutura básica de um frame com espaçamento entre subportadoras de $15 \mathrm{KHz}$ no domínio do tempo e frequência

tamanho da constelação do MCS $j$ e $T_{s}$ o tempo do símbolo, a taxa de bits de um bloco de recursos $r^{(j)}$ alcançada para o MCS $j$ é dada por:

$$
r^{(j)}=\frac{R_{j}^{(c)} \log _{2}\left(M_{j}\right)}{T_{s} N_{s}} \sum_{s=1}^{N_{s}} N_{s c}^{d}(s) .
$$

O CQI (Channel Quality Indicator, Indicador de Qualidade do Canal) é informado pelo usuário ou pode ser estimado por meio de algoritmos de estimação de canal, a fim de não sobrecarregar o canal de uplink. A partir do CQI são definidas a taxa de código e o esquema de modulação, ou seja, a informação de qual MCS deve ser adotado para o usuário $k$ no bloco de recurso $n$. Neste artigo, a taxa de código e o índice MCS associados com a SINR (Signal-to-Interference-plus-Noise-Ratio, Relação Sinal-Ruído e Interferência) são determinados através do mapeamento da tabela de CQI de 4 bits conforme [3GPP 2016].

Cada SB é alocado a apenas um usuário em um TTI. Seja $q_{n}(i) \in\{1,2, \ldots, K\}$ o usuário alocado no bloco de recurso $n$ no TTI $i$. A taxa de bits nesse bloco de recurso depende do MCS do usuário, logo a taxa de bits do mesmo bloco de recurso pode ser diferente para cada usuário. Alocar os blocos de recursos aos usuários com maiores taxas de bits tende a aumentar a utilização da rede.

Seja $x(t)=\left[x_{1}(t), x_{2}(t), \ldots, x_{N}(t)\right]$ o vetor de tamanho $N$, composto pelos elementos $x_{n}(t), n \in N$, que associa cada bloco de recurso a um usuário no instante de tempo $t$, por exemplo, se $x_{1}(t)=2$, o bloco de recurso 1 está alocado para o usuário 2 no instante de tempo $t ; m_{k, n}(t)$ o MCS adotado para o usuário $k$ no bloco de recurso $n$ no instante de tempo $t$, a taxa de bits do usuário $k, r_{k}$, no instante de tempo $t$ é dada por:

$$
r_{k}(t)=\sum_{n=1}^{N} I(x[n]=k) r^{\left(m_{k, n}(t)\right)},
$$

onde $I(x[n]=k)$ é 1 se $x[n]=k$ e 0 caso contrário. A taxa de bits total $T_{b}$ do 
sistema é:

$$
T_{b}(t)=\sum_{k=1}^{K} r_{k}(t) .
$$

Maximizar a taxa de bits total do sistema $T_{b}$ é uma forma de melhorar a utilização da rede. Porém, juntamente com o aumento da taxa de bits total do sistema é necessário atender certos requisitos de banda de cada usuário. Assim, tem-se um problema de otimização que consiste em maximizar a taxa do sistema atendendo a taxa mínima de cada usuário:

$$
(x): \max T_{b},
$$

sujeito a:

$$
r_{k} \geq R_{k} \forall k
$$

\section{Algoritmo de Alocação de Blocos de Recurso Proposto}

Em [Ferreira et al. 2015], é proposto um algoritmo de escalonamento que procura minimizar o retardo em redes sem fio. A principal diferença do algoritmo apresentado em [Ferreira et al. 2015] e do algoritmo proposto neste artigo é que neste último o limitante de retardo é estimado a medida que as características dos dados de tráfego no sistema variam, utilizando conceitos de Cálculo de Rede, conforme explicado na Seção 2. No algoritmo apresentado em [Ferreira et al. 2015] o retardo foi calculado com base em valores reais passados de retardo da rede. Sendo assim, a vantagem da proposta do presente artigo é que o algoritmo pode tomar decisões antecipadas utilizando as estimativas e previsões de retardo baseados em uma modelagem do tráfego e do sistema.

O algoritmo proposto pode ser resumido em três fases:

1. Estima o número de SBs requeridos para cada usuário com prioridade baseada no ganho médio de canal;

2. Aloca os SBs para os usuários de acordo com a prioridade, o limitante de retardo estimado, calculado conforme Equação (3), e o critério de retardo máximo definido;

3. Aloca os SBs remanescentes para os usuários com prioridade definida de acordo com o retardo estimado.

A prioridade na alocação dos recursos é definida em ordem crescente pelo ganho médio do canal por usuário, ou seja, os usuários com piores condições de canal tem maior prioridade. O ganho médio do canal $G_{k}$ por usuário $k$ é calculado pela seguinte equação:

$$
G_{k}=\frac{1}{N} \sum_{n=1}^{N} g_{k, n}
$$

onde $g_{k, n}$ é o ganho médio do canal para o usuário $k$ no $n$-ésimo SB e $N$ é o número de SBs disponíveis para downlink.

A quantidade $N_{k}$ de SBs requeridas para cada usuário $k$ é calculada da seguinte forma, com base nas condições do canal:

$$
N_{k}=\operatorname{round}\left(\left(\frac{G_{k}}{G_{1}+G_{2}+\ldots+G_{k}}\right) * N\right) \text {, }
$$


onde $G_{k}$ é o ganho médio do canal por usuário $k, N$ é o número de SBs disponíveis para downlink e round(.) é uma função de arredondamento para o inteiro mais próximo.

Após calculado a prioridade de alocação, os SBs com maior CQI são alocados de acordo com a quantidade de SBs estimadas para cada usuário. Depois é estimado o limitante de retardo conforme Equação (3) e verificado se o critério de retardo máximo é satisfeito. Se o critério não é satisfeito o algoritmo continua alocando SBs com maior CQI até satisfazer o critério.

O algoritmo garante a alocação dos SBs de forma justa, uma vez que prioriza os usuários com piores condições do canal com objetivo de satisfazer o critério de retardo, ao mesmo tempo que aloca uma quantidade maior de SBs para os usuários com melhores condições de canal.

Após verificado se o critério de retardo máximo foi satisfeito para todos os usuários, o algoritmo aloca os SBs remanescentes, se houver, priorizando o valor de retardo estimado para cada usuário, ou seja, os usuários com maior valor de retardo tem maior prioridade. O objetivo é reduzir o retardo médio depois de satisfeito o critério.

\section{Simulações e Resultados}

Nesta seção são apresentados os resultados das simulações do algoritmo de alocação proposto, comparando com o algoritmo PSO, o algoritmo QoS guaranteed [Guan et al. 2011] e o algoritmo Min-delay [Ferreira et al. 2015].

O algoritmo denominado de PSO (Particle Swarm Optimization) [Su et al. 2012] neste trabalho consiste na aplicação do algoritmo de enxame de partículas para solução do problema de otimização representado pelas Equações (8) e (9). Já os algoritmos QoS guaranteed [Guan et al. 2011] e Min-delay [Ferreira et al. 2015] buscam reduzir a complexidade computacional do problema de otimização representado pelas Equações (8) e (9), levando em conta, respectivamente, a restrição da taxa mínima requerida e o critério de retardo máximo utilizando a informação de retardo real da rede.

As simulações foram realizadas por meio do software MATLAB versão R2018a, utilizando um microcomputador com a seguinte configuração: Processador Intel Core I7-4785T CPU 2.20GHz, 8GB RAM, HD SATA III 7200 RPM, Windows 10 64bits.

\subsection{Modelo de canal e parâmetros do sistema}

As condições do canal para cada usuário e SB em termos de SINR foram gerados para 1000 TTIs através da seguinte equação [Ni et al. 2013]:

$$
S I N R=\frac{G_{o} P_{o}}{\sum_{j=1}^{J} G_{j} P_{j}+\sigma_{n}^{2}},
$$

onde $G_{o}$ é o ganho do canal para a potência de transmissão $P_{o}, G_{j}$ é o ganho do canal para os sinais de interferência com potência $P_{j}, \sigma_{n}^{2}$ é a potência do ruído e $J$ é o número de células de interferência. 
Tabela 1. Parâmetros de simulação para o sistema de rede sem fio e modelagem de canal

\begin{tabular}{|c|c|}
\hline Parâmetro & Valor \\
\hline Largura de banda & $3 \times 396 \mathrm{MHz}$ \\
\hline Número de RBs por portadora & 275 \\
\hline Número de TTIs simulados & 1000 \\
\hline Taxa mínima requerida & Média do tráfego \\
\hline Retardo máximo & $60 \mathrm{kB}$ \\
\hline Tamanho de buffer por usuário & $120 \mathrm{KHz}$ \\
\hline Espaçamento entre subportadoras & CDLSK, 16-QAM, 64 -QAM e 256-QAM \\
\hline Modulações & $0.5 \mathrm{~km}$ \\
\hline Modelo multipercurso & $-174 \mathrm{dBm} / \mathrm{Hz}$ \\
\hline Distância entre UE e BS & $35 \mathrm{dBm}$ \\
\hline Densidade de potência do ruído branco & $15 \mathrm{dBi}$ \\
\hline Potência máxima do transmissor BS & $0 \mathrm{dBi}$ \\
\hline Ganho da antena BS & $9 \mathrm{~dB}$ \\
\hline Ganho da antena UE & $4 \mathrm{~dB}$ \\
\hline Figura de ruído UE & $3 \mathrm{~km} / \mathrm{h}$ \\
\hline Margem de interferência UE & \\
\hline Velocidade UE & CDS \\
\hline
\end{tabular}

A taxa de bit e o índice MCS associado ao SINR são definidos com CQI de 4 bits conforme [3GPP 2016]. A Tabela 1 apresenta os parâmetros de transmissão downlink no sistema e o modelo de canal considerado, com valores baseados nos valores utilizados na literatura [Santos Jr and Vieira 2015] [Guan et al. 2011] [Su et al. 2012] [3GPP 2011] [3GPP 2017] [3GPP 2018b] [3GPP 2018a] [3GPP 2019]. Foi considerado o modelo multipercurso CDL-A (Clustered Delay Line - A, Linha de Atraso Agrupada - A) para modelagem do canal por ser mais adequado para simulação de cenários de propagação com ondas milimétricas, conforme normativas mais recentes do órgão 3GPP [3GPP 2017].

Foram consideradas cinco séries reais de tráfego TCP/IP (Transmission Control Protocol/Internet Protocol, Protocolo de Controle de Transmissão/Protocolo Internet) para representação dos usuários em 1000 TTIs de forma aleatória durante a simulação, agregadas no domínio do tempo em intervalos de $1 \mathrm{~ms}$. As séries de tráfego consideradas representam o tráfego TCP/IP entre a Universidade de Waikato com redes externas, coletados entre 20/05/2011 e 29/10/2011 (disponíveis no endereço http://wand.net.nz/wits/waikato/8/).

\subsection{Resultados}

Considerando os parâmetros de transmissão downlink e do modelo de canal dados na Tabela 1, avalia-se nesta seção o desempenho do algoritmo de escalonamento proposto em comparação com outros da literatura.

Nas simulações apresentadas nesta seção, foram considerados para o algoritmo PSO os parâmetros utilizados no cenário descrito em [Su et al. 2012]: 30 


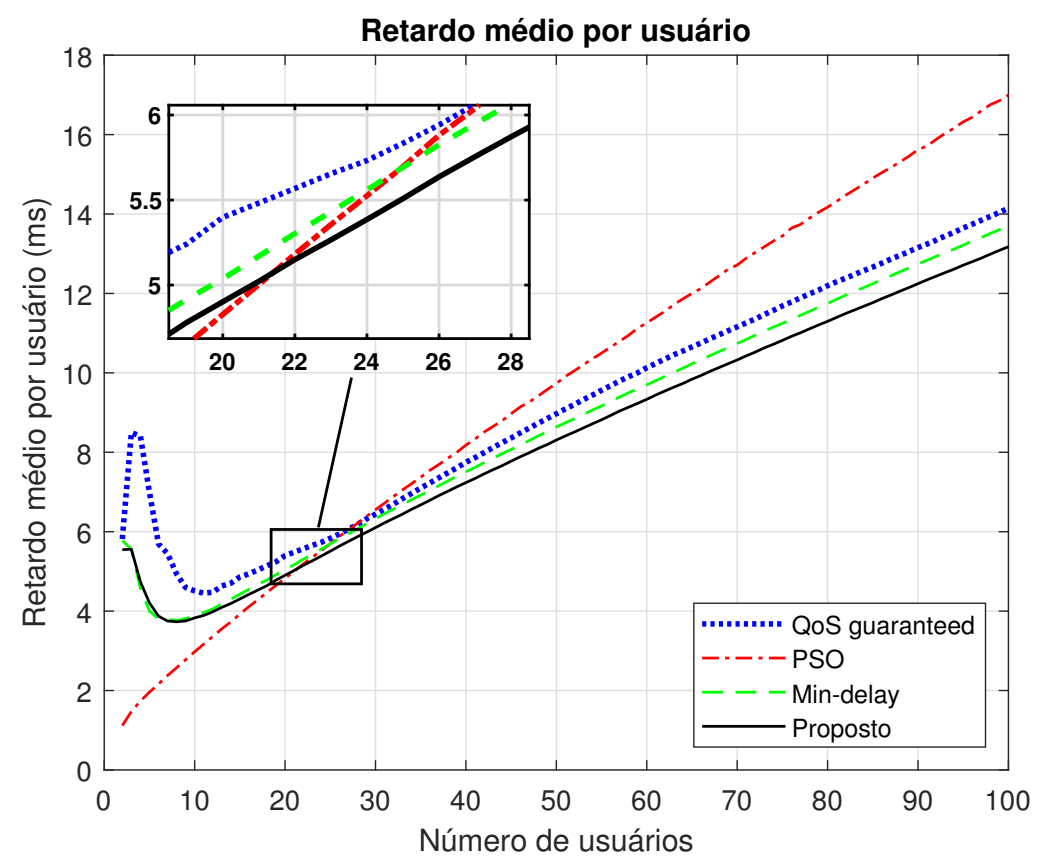

Figura 3. Retardo médio em função do número de usuários

indivíduos, 100 iterações como critério de parada, peso de inércia $w=\frac{(100-i)}{100}$, sendo $i$ o número de iterações, $c_{1}=c_{2}=2$.

Na Figura 3 é apresentado o retardo médio por usuário. Verifica-se que os valores são significativamente menores para o algoritmo proposto do que os valores dos algoritmos QoS guaranteed e PSO se considerado mais de 22 usuários em simulação. Este fato mostra que em cenários de maior densidade de número de usuários o algoritmo proposto apresenta desempenho superior aos demais algoritmos em termos de retardo na rede.

O algoritmo PSO apresenta maiores valores de vazão total se considerado até 25 usuários, conforme pode ser visto na Figura 4. Este fato era esperado visto o algoritmo PSO ser uma heurística de maximização da vazão total em detrimento do elevado tempo de processamento. Porém o desempenho do algoritmo PSO decai para mais de 25 usuários, devido a sua função objetivo que considera o critério de taxa mínima requerida. O algoritmo proposto apresenta valores de vazão total similares aos valores apresentados pelo algoritmo Min-delay e maiores do que os valores apresentados pelo algoritmo QoS guaranteed.

Quanto ao tempo de processamento, o algoritmo proposto apresenta valores bastante inferiores aos apresentados pelo algoritmo PSO e superiores aos valores apresentados pelos algoritmos QoS guaranteed e Min-delay, conforme pode ser visto na Figura 5.

O maior tempo de processamento do algoritmo PSO comparado aos dos outros algoritmos considerados pode ser explicado devido a sua maior complexidade computacional. A complexidade computacional do algoritmo baseado na heurística PSO deve ser analisada considerando os laços, ou loops, para busca da melhor 


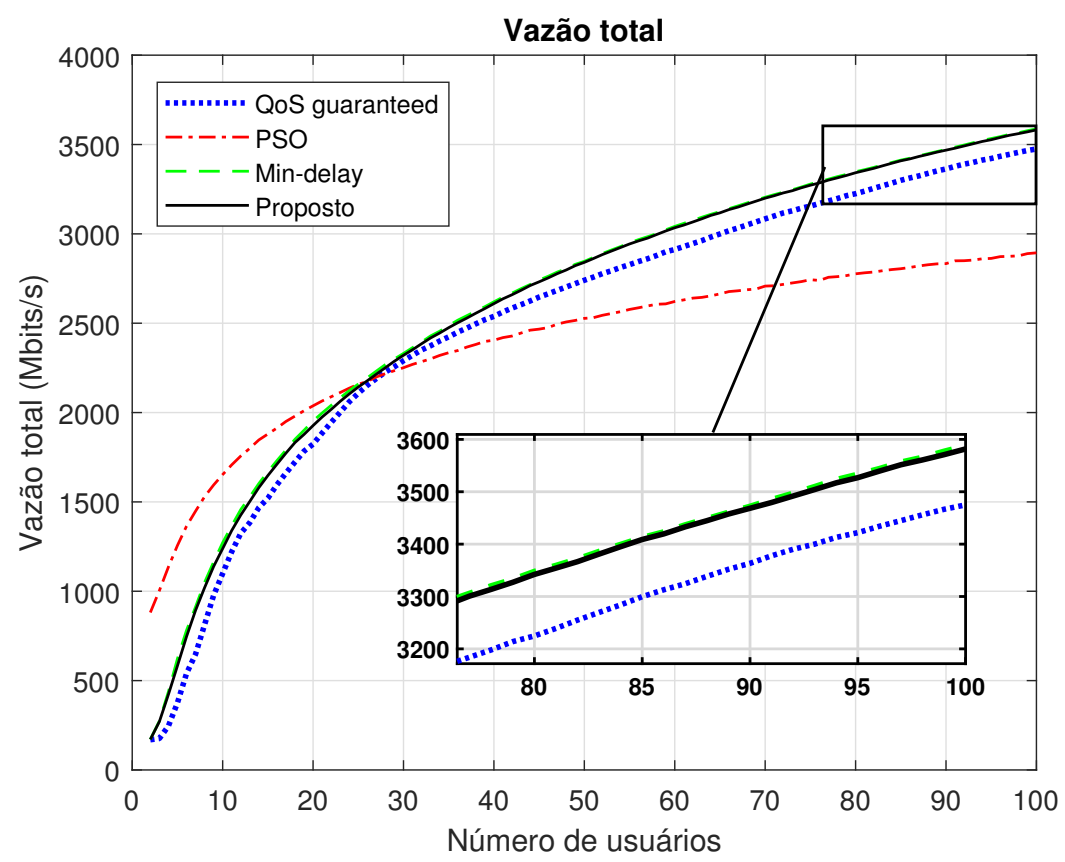

Figura 4. Vazão total em função do número de usuários

solução. Estes laços são definidos pelo número de iterações maxit, o tamanho da população $P_{o}$ e o tamanho da matriz de dados de entrada $N \times K$, sendo $N$ a quantidade de blocos de escalonamento disponíveis e $K$ a quantidade de usuários no sistema. Assim, a complexidade computacional do algoritmo do escalonador baseado em PSO é igual a $O\left(N \times K \times P_{o} \times\right.$ maxit $)$.

Em relação à complexidade computacional dos algoritmos QoS guaranteed, Min-delay e do algoritmo proposto, considera-se a quantidade de blocos de escalonamento da rede $N$ e o número de usuários $K$ apenas, uma vez que não são aplicadas heurísticas de otimização nestes algoritmos. A alocação nestes cenários se baseia apenas na estimativa do número de blocos e na verificação dos parâmetros de QoS, ambos com complexidade $O(1)$. A complexidade destes algoritmos é definida então pelo número de execuções de alocação de blocos em função do número de usuários $K$ e quantidade de blocos $N$, ou seja, a complexidade é $\leq O(N \times K)$.

O índice de justiça (fairness) é uma importante medida para determinar se os usuários estão recebendo uma distribuição justa dos recursos do sistema. A Figura 6 mostra que o algoritmo proposto apresenta os maiores valores de fairness se considerado mais de 40 usuários em simulação, mostrando que o algoritmo distribui seus recursos de forma justa em cenários com maior densidade de usuários. O índice fairness foi calculado da seguinte forma [Jain et al. 1998]:

$$
\text { Fairness }=\frac{\left[\sum_{k=1}^{K} x_{k}\right]^{2}}{K \sum_{k=1}^{K} x_{k}^{2}},
$$

onde $x_{k}=\frac{r_{k}}{R_{k}}$ é a taxa normalizada, $r_{k}$ é a taxa do usuário $k, R_{k}$ é a taxa mínima requerida do usuário $k$ e $K$ é o número total de usuários. 


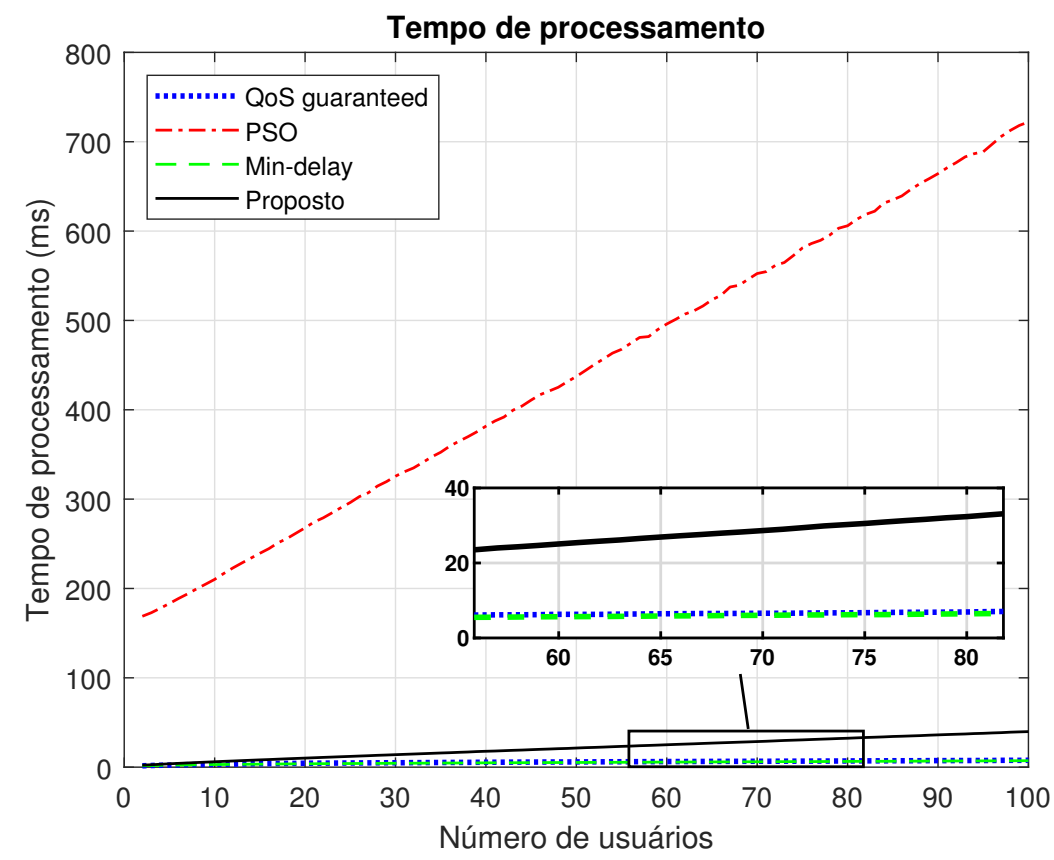

Figura 5. Tempo de processamento em função do número de usuários

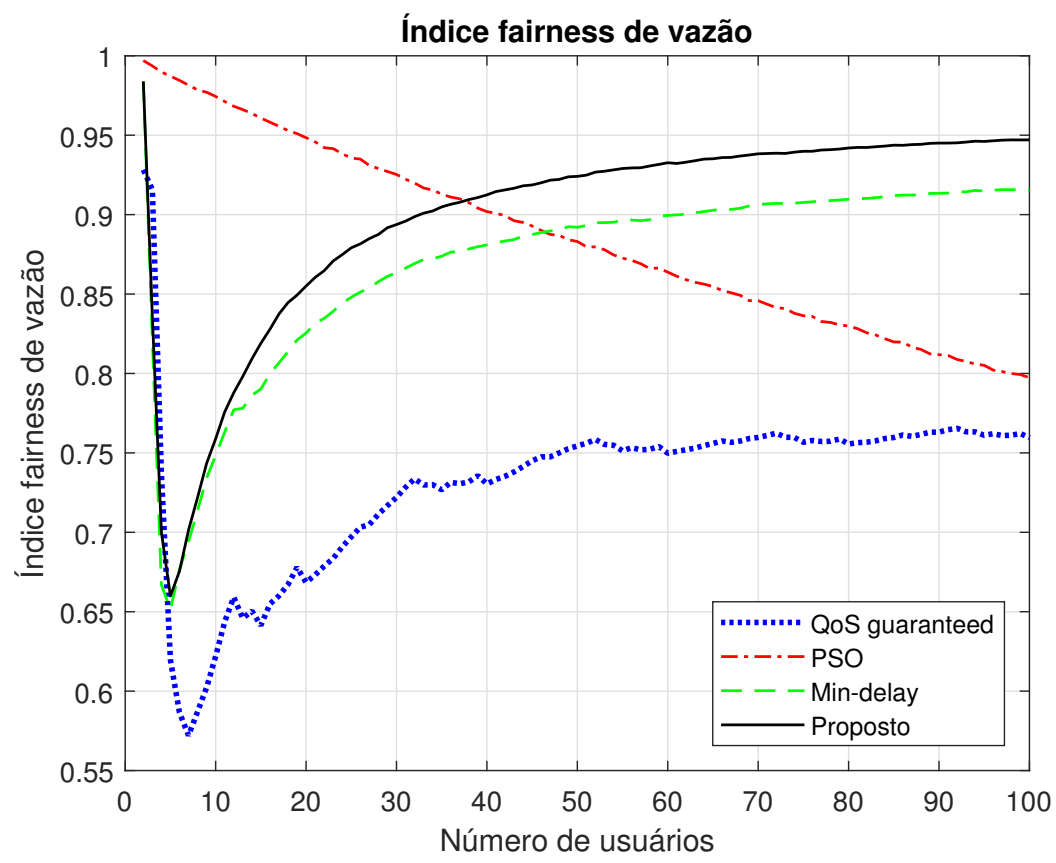

Figura 6. Índice de justiça (fairness) em função do número de usuários 
Tabela 2. Taxa de perda de bits (\%) em função do número de usuários

\begin{tabular}{|l|c|c|c|c|}
\hline \multirow{2}{*}{ Algoritmo } & \multicolumn{4}{|c|}{ Número de usuários } \\
\cline { 2 - 5 } & 25 & 50 & 75 & 100 \\
\hline QoS guaranteed & 0 & 0 & 0 & 0 \\
\hline PSO & 0 & 0.0078 & 0.2489 & 0.9690 \\
\hline Min-delay & 0 & 0 & 0 & 0 \\
\hline Proposto & 0 & 0 & 0 & 0 \\
\hline
\end{tabular}

A Tabela 2 mostra que o algoritmo PSO apresenta os maiores valores de perda de bits se considerado até 100 usuários em simulação.

\section{Conclusão}

Neste artigo é proposto um algoritmo de alocação de blocos de recurso em redes sem fio cujo objetivo é utilizar a informação de retardo estimado por Cálculo de Rede através de conceitos de curva de serviço e processo envelope MFBAP a fim de reduzir o retardo do sistema. Assim, pode-se atualizar a estimativa de retardo a medida que as características dos dados de tráfego no sistema variam e tomar decisões antecipadas sobre a alocação de recursos.

Os resultados das simulações mostram que o desempenho do algoritmo proposto é em geral superior ou similar ao desempenho dos algoritmos QoS guaranteed [Guan et al. 2011], PSO [Su et al. 2012] e Min-delay [Ferreira et al. 2015], principalmente se considerado um cenário com maior número de usuários na rede, ou seja, cenários mais próximos da realidade dos cenários de comunicações móveis.

Em termos de vazão, o algoritmo proposto provê os maiores valores se considerado mais de 25 usuários em simulação. Em relação ao retardo médio, o algoritmo proposto apresenta os menores valores em geral. Nota-se também que o algoritmo apresenta baixo tempo computacional, valores de fairness acima de 0.9 se considerado mais de 30 usuários na rede, maiores do que os valores apresentados pelos demais algoritmos, e apresenta baixa taxa de perda.

Como proposta para pesquisa futura, pretende-se calcular uma curva de serviço específica para o escalonador proposto de forma a melhorar a estimativa de limitante de retardo.

\section{Agradecimentos}

Os autores gostariam de agradecer à FAPEG (Fundação de Amparo à Pesquisa no Estado de Goiás) pelo apoio no desenvolvimento da pesquisa.

\section{Referências}

3GPP (2008). 3gpp ts 36.104 version 8.3.0 release 8. lte; evolved universal terrestrial radio access (e-utra); base station (bs) radio transmission and reception.

3GPP (2011). 3gpp tr 36.931 version 9.0.0 release 9. 1te; evolved universal terrestrial radio access (e-utra); radio frequency (rf) requirements for lte pico node b. 
3GPP (2016). 3gpp ts 36.213 version 13.0.0 release 13. lte; evolved universal terrestrial radio access (e-utra); physical layer procedures.

3GPP (2017). 3gpp tr 38.901 version 14.0.0 release $14.5 \mathrm{~g}$; study on channel model for frequencies from 0.5 to $100 \mathrm{ghz}$.

3GPP (2018a). 3gpp ts 38.104 version 15.2 .0 release $15.5 \mathrm{~g}$; nr; base station (bs) radio transmission and reception.

3GPP (2018b). 3gpp ts 38.211 version 15.2 .0 release $15.5 \mathrm{~g}$; nr; physical channels and modulation.

3GPP (2019). 3gpp ts 38.306 version 15.7.0 release $15.5 \mathrm{~g}$; nr; user equipment (ue) radio access capabilities.

Delgado, O. and Jaumard, B. (2010). Scheduling and resource allocation in lte uplink with a delay requirement. In 2010 8th Annual Communication Networks and Services Research Conference, pages 268-275.

Ferreira, M. V. G., Vieira, F. H. T., and Abrahão, D. C. (2015). Minimizing delay in resource block allocation algorithm of lte downlink. In 2015 International Workshop on Telecommunications (IWT), pages 1-7.

Guan, N., Zhou, Y., Tian, L., Sun, G., and Shi, J. (2011). Qos guaranteed resource block allocation algorithm for lte systems. In 2011 IEEE 7th International Conference on Wireless and Mobile Computing, Networking and Communications (WiMob), pages 307-312.

Jain, R., Chiu, D.-M., and Hawe, W. (1998). A quantitative measure of fairness and discrimination for resource allocation in shared computer systems. CoRR, cs.NI/9809099.

Le Boudec, J.-Y. and Thiran, P. (2004). Network calculus: A theory of deterministic queuing systems for the internet. 2050.

Ni, M., Xu, X., and Mathar, R. (2013). A channel feedback model with robust sinr prediction for lte systems. In 2013 7th European Conference on Antennas and Propagation (EuCAP), pages 1866-1870.

P, M., Gadre, V., and Desai, U. (2003). Multifractal Based Network Traffic Modeling. Springer US.

Santos Jr, J. and Vieira, F. H. (2015). Estimation of backlog and delay in ofdm/ tdma systems with traffic policing through network calculus. Latin America Transactions, IEEE (Revista IEEE America Latina), 13:796-803.

Su, L., Wang, P., and Liu, F. (2012). Particle swarm optimization based resource block allocation algorithm for downlink lte systems. In 2012 18th Asia-Pacific Conference on Communications (APCC), pages 970-974.

Vieira, F., Costa, V., Ling, L., and Rocha, F. (2016). Estimação de Probabilidade de Transbordo do Buffer em Redes OFDM-TDMA utilizando Cadeias de Markov e Curva de Serviço. TEMA (São Carlos), 17:3 - 20. 